\title{
Localization in disordered potential in photonic lattice realized in time domain
}

Ilya D. Vatnik, Alexey M. Tikan, Dmitry V. Churkin, Andrey A. Sukhorukov

Ilya D. Vatnik, Alexey M. Tikan, Dmitry V. Churkin, Andrey A. Sukhorukov, "Localization in disordered potential in photonic lattice realized in time domain," Proc. SPIE 10457, AOPC 2017: Laser Components, Systems, and Applications, 1045733 (24 October 2017); doi: 10.1117/12.2285481

SPIE. Event: Applied Optics and Photonics China (AOPC2017), 2017, Beijing, China 


\title{
Localization in disordered potential in photonic lattice realized in time domain
}

\author{
Ilya D. Vatnik ${ }^{\mathrm{a} *}$, Alexey M. Tikan ${ }^{\mathrm{a}}$, Dmitry V. Churkin ${ }^{\mathrm{a}}$, Andrey A. Sukhorukov ${ }^{\mathrm{b}}$ \\ ${ }^{a}$ Novosibirsk State University, Pirogova str. 2, Novosibirsk 630090, Russia; \\ ${ }^{b}$ Nonlinear Physics Centre, Research School of Physics and Engineering, The Australian National \\ University, Canberra, ACT 2601, Australia
}

\begin{abstract}
We describe theoretically and realize experimentally Anderson localization for optical pulses in time domain, using a photonic mesh lattice with random phase modulation implemented with coupled optical fiber loops. We demonstrate that strongest degree of localization is limited and increases in lattices with wider band-gaps.
\end{abstract}

Keywords: synthetic photonic lattice, disorder, localization, photonic lattice, mesh lattice

\section{INTRODUCTION}

A synthetic photonic lattice is a full analogue of a photonic mesh lattice but realized in time domain rather than in space domain. The synthetic photonic lattice is the optical-fiber based system, comprising two fiber loops that are coupled via a directional coupler. These two fiber loops differ in length. Thus a single pulse launched into the system produces a train of pulses circulating, interfering and multiplying within the system. It can be shown that that time dynamics of light pulses is equivalent to the evolution of light beams expected in the spatial configurations of a mesh lattice. Evolution of a pulse chain depends on phase of each pulse acquired during the roundtrip and can be controlled in an experiment by phase modulators. Here phase modulation plays role of an optical potential in the system. Apart from the conventional mesh photonic lattices, this optical potential can be easily and precisely controlled by means of an electro-optical modulator together with electrical waveform generator. Up to date, a number of different phenomena including random walk of a single particle ${ }^{1}$, localization on a defect ${ }^{2}$, Bloch oscillations and PT symmetry breaking ${ }^{3}$ and solitons ${ }^{4}$ were realized in this system.

It's well known that in systems described by the Schrodinger equation a disordered potential leads to the appearance of localized states, that is Anderson localization effect ${ }^{5}$. Firstly predicted for electrons in non-ideal crystals, the phenomenon was successfully established and then experimentally realized in a number of photonic structures including one-, two- and three-dimensional photonic lattices ${ }^{6-8}$, disordered fibers ${ }^{9,10}$. In all this realizations localization occurred in space domain. We consider an analog of the Anderson localization in a synthetic photonic lattice that is obtaining a chain of pulses localized at a small number of sites in the coordinate $\mathrm{n}$ (localized in time) due to the introduction of a potential random over $\mathrm{n}$. It should be noted that early observation of Anderson localization was implemented previously in a system that conceptually was similar to synthetic photonic lattices. In this system a set of two polarizations effectively play a role of two different loops. However, only the regime of strong disorder was realized ${ }^{11}$, while the effect of localization should occur in one-dimensional systems regardless the potential strength ${ }^{12,13}$. Here we show that it does happen for any amount of disorder. Then we make a theoretical study revealing the properties of pulse trains circulating within the synthetic photonic lattice with disorder, and present experimental realization of the phenomenon as well as discussion on effects limiting experiment performance.

\section{NUMERICAL SIMULATIONS}

The system is described by a set of equations for the amplitudes of light pulse in the short (Um n ) and long (Vm,n ) loops.

AOPC 2017: Laser Components, Systems, and Applications, edited by Shibin Jiang, Lijun Wang,

Lan Jiang, Long Zhang, Proc. of SPIE Vol. 10457, 1045733 - (c) 2017 SPIE

CCC code: $0277-786 \mathrm{X} / 17 / \$ 18 \cdot$ doi: $10.1117 / 12.2285481$ 


$$
\begin{gathered}
U_{n}^{m+1}=\exp \left(i \varphi_{n}\right) \frac{1}{\sqrt{2}}\left[U_{n+1}^{m}+i V_{n+1}^{m}\right] \\
V_{n}^{m+1}=\frac{1}{\sqrt{2}}\left[V_{n-1}^{m}+i U_{n-1}^{m}\right]
\end{gathered}
$$

Here $\mathrm{m}$, roundtrip number, is discrete time coordinate and $\mathrm{n}$ is a space-like coordinate defined by particular position of a pulse within the loop.

Random potential was realized by applying a random phase shift in the short loop. We characterized the random potential by the amplitude of the random potential $\varphi_{\max }$, which varies from zero to $\pi$. Based on the model developed previously ${ }^{14}$, the time evolution of the wave packet was calculated for different values of the amplitude of the random potential. As shown by the calculations, the presence of a disordered potential indeed leads to the localization of the wave packet $\left|u_{n}^{m}\right|$ in time. The larger the amplitude of the random potential, the stronger the localization effect (Fig. 1).

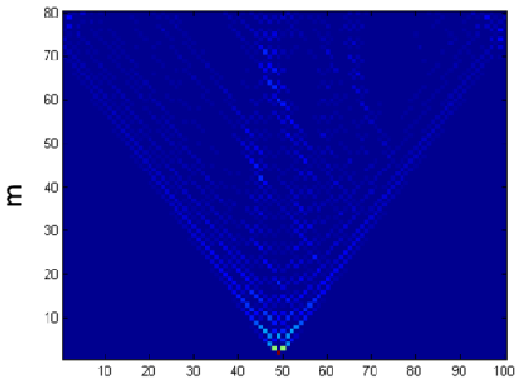

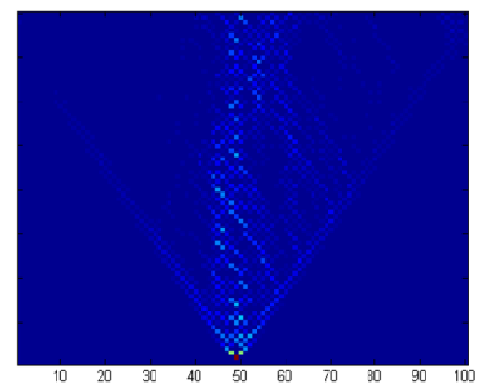

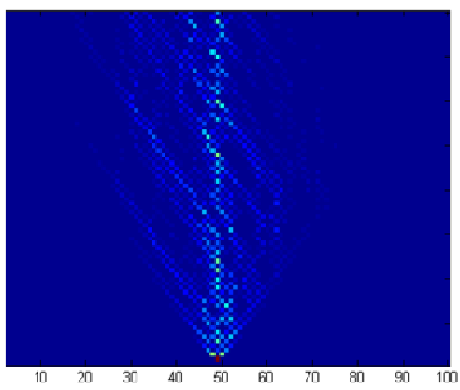

Figure 1. Evolution of the envelope of the amplitude of a chain of pulses circulating in a synthetic photonic lattice with a random potential. The results of numerical calculation for realization of a random potential of depth $0.2 \pi$ (left panel), $0.4 \pi$ (central panel), $0.6 \pi$ (right panel) are presented. Here $n$ is a number of a slot, $m$ is a roundtrip number. The calculation assumes that at the initial moment of time a single pulse is put into the $50^{\text {th }}$ slot.

For quantitative characterization of the degree of localization, a standard measure of localization, participation ratio $P(m)=\left(\sum_{n}\left|U_{n}^{m}\right|^{2}\right)^{2} / \sum_{n}\left|U_{n}^{m}\right|^{4}$, was calculated. The wider the envelope of the pulse chain for a certain $\mathrm{m}$ the larger the value of the participation ratio. As the strength of the disorder increases, the participation ratio decreases (Fig. 2a), which is a manifestation of localization.
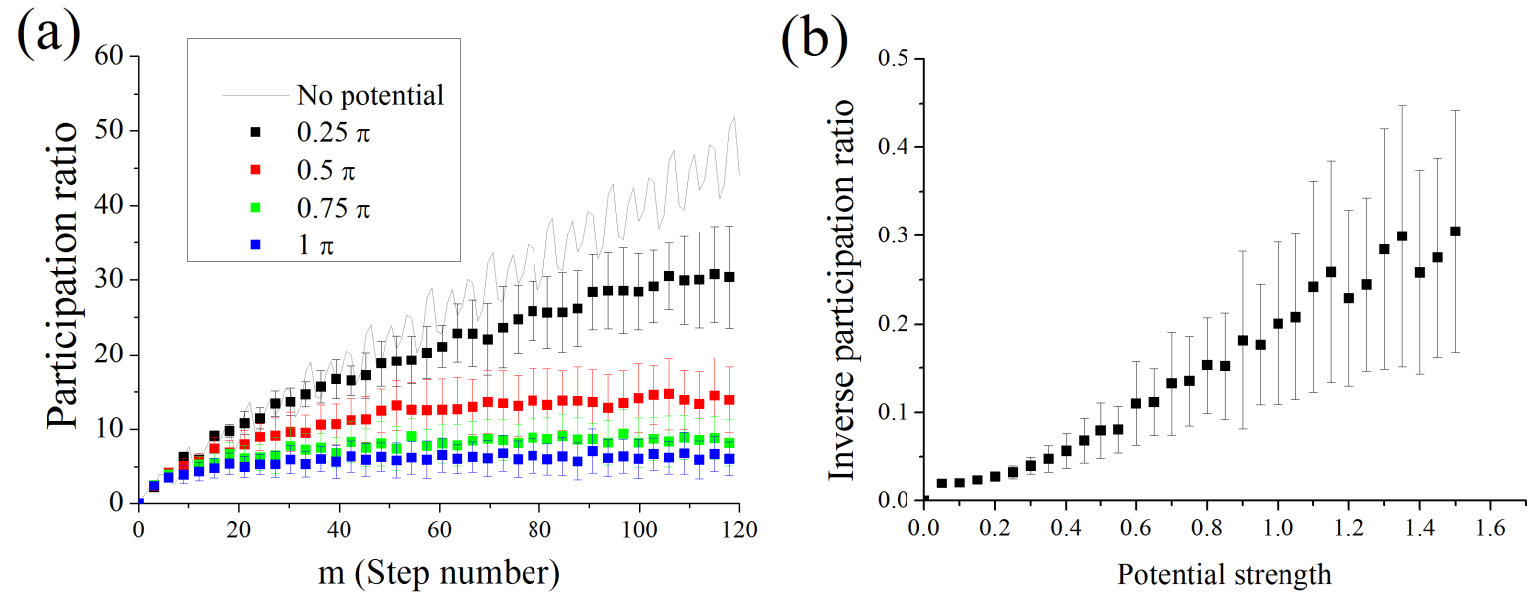

Figure 2. (a) The participation ratio $\mathrm{P}(\mathrm{m})$ for the case without potential (solid gray curve) and for a random potential of different strength (colors show the data for different amplitudes of the random potential). As the potential strength increases, localization is observed, characterized by the fact that the packet of pulses occupies a smaller number of slots. $(\mathrm{b})$ The inverse participation number $(1 / \mathrm{P}(\mathrm{m}=$ 100)) and its fluctuations, calculated as the root-mean-square deviation for an ensemble of 100 realizations. With an increase in the strength of the disorder, a linear growth of both the magnitude of the inverse participation number and its fluctuations happen. 
The participation ratio undergoes statistical fluctuations due to deviations for different realizations of a random potential. The increase in the degree of fluctuations of the participation ratio $1 / \mathrm{P}(\mathrm{m}=100)$ with increase of strength of the disorder is a distinguishing feature of the Anderson localization ${ }^{15}$. We analyzed fluctuations of the inverse participation ratio as a function of the strength of the disorder calculated over 100 different realizations of the random potential and showed that such fluctuations increase with increasing degree of disorder (Fig. 2b), which is a proof of localization in the system under consideration.

A feature of the Anderson localization in 1D is the fact that the localization occurs at an arbitrarily small potential. We tested this property for the system. For this purpose, the evolution of the chain of pulses was calculated for very long propagation times $(\mathrm{m})$, and the participation ratio was calculated for a small disorder value. Smallness can be established by comparison with the phase acquired during the passage through the coupler $(\pi / 4)$. We have found that the participation ratio is saturated at large evolution times (of the order of 1000 roundtrips), that is a proof of the presence of localization (Figure 3).

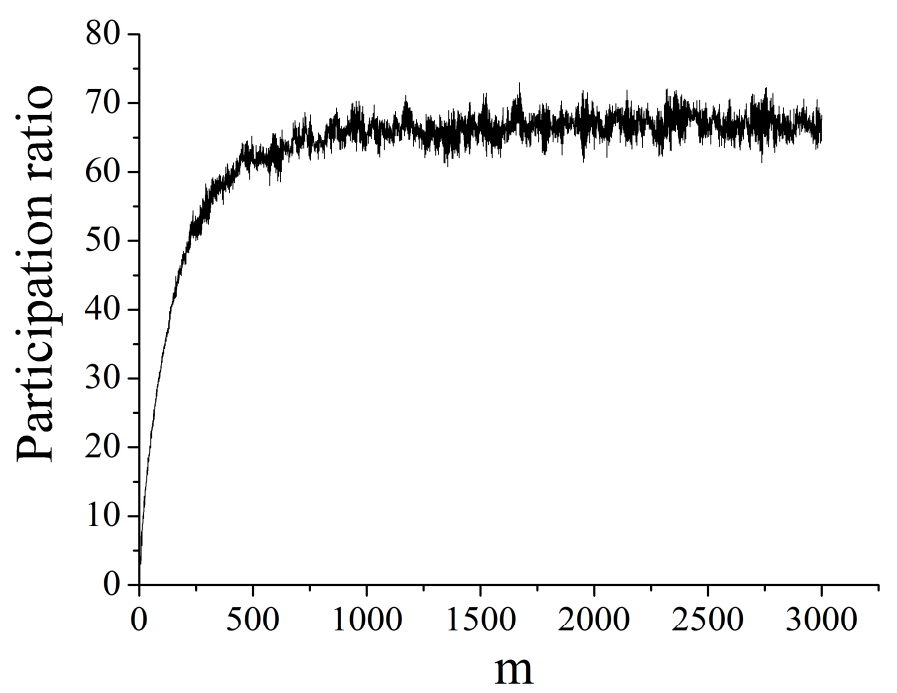

Figure 3. The participation ratio $\mathrm{P}(\mathrm{m})$ for the case of a weak potential $\left(\varphi_{\max }=0.2 \pi\right)$. The curve asymptotically approaches the stationary value. Localization occurs at roundtrip numbers of the order of 1000.

We also analysed another characteristic - the wave function distribution form for those values of $\mathrm{m}$ when $\mathrm{P}(\mathrm{m})$ is saturated. It was found that the quantity $\left\langle\left|U_{n}^{m}\right|^{2}>\right.$ averaged over the ensemble of realizations has an exponential form (see Fig. 4). This fact is yet another proof of the existence of Anderson localization.

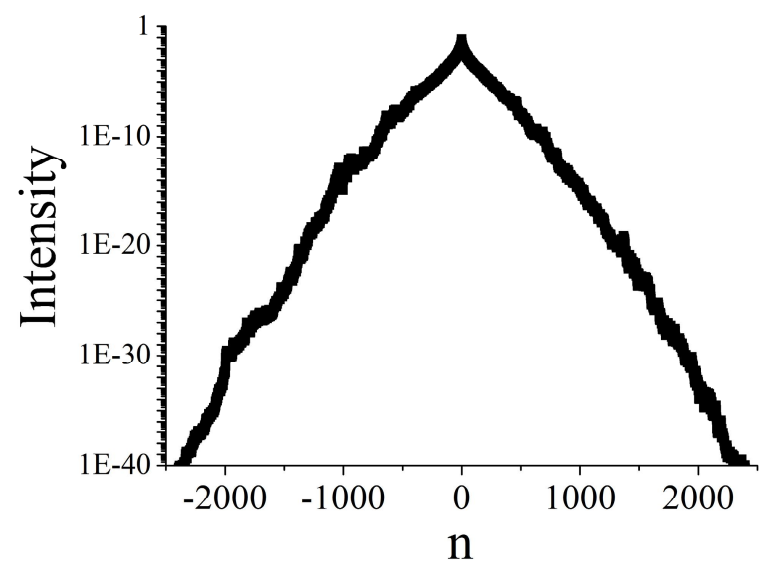

Figure 4. $\left\langle\left|U_{n}^{m=4000}\right|>\right.$ as a function of slot number $\mathrm{n}$ in the logarithmic scale. Random potential strength is $\varphi_{\max }=\pi / 2$. Aaveraging has been done over 180 realizations. 
Finally, the mode structure and the dispersion law for eigenmodes was studied using the method described previously ${ }^{14}$. In the trivial case with no potential there is a band-gap in the dispersion law. As the strength of the random potential increases, the width of the band gap decreases, and for some potential parameters (namely, $\varphi_{\max }=2 \pi$ ), it can become equal to zero (Fig. 5a).

Despite the fact that a weak random potential does not practically change the dispersion law, it leads to the appearance of localized modes (Figure 5b), which agrees with the statement that there is no Anderson transition in one-dimensional systems. The modes located at the edge of the forbidden band are more localized.
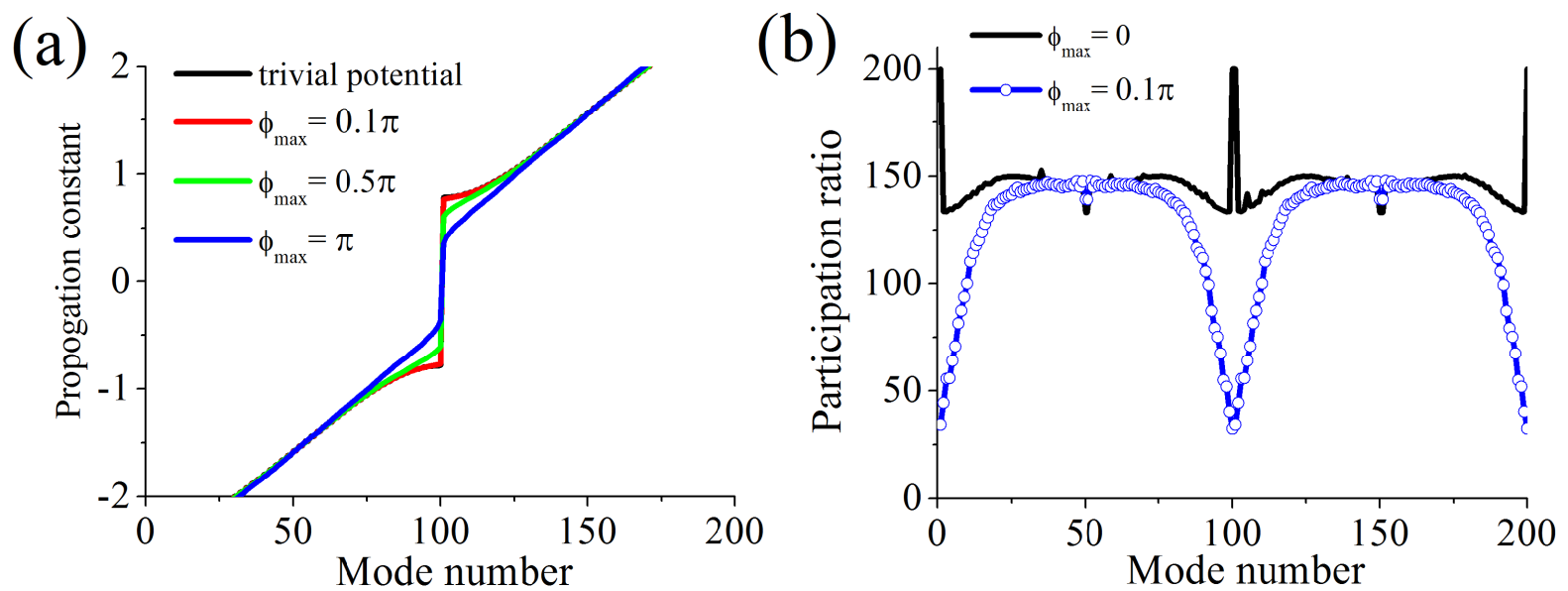

Figure 5. (a) The dispersion relation for a synthetic photon lattice with a random potential of different strengths. The width of the band gap decreases with increasing strength of the disorder. (b) The participation number $\mathrm{P}(\mathrm{m})$ for different eigenmodes of a synthetic photonic lattice without a potential (black line) and with a random potential of small strength (blue symbols). The presence of a weak potential leads to the localization of eigenmodes located at the boundaries of the band gap.

\section{EXPERIMENTAL REALIZATION}

Basing on the analysis carried out in the previous section, an experiment was performed to experimentally observe the Anderson localization. The scheme of the experimental setup is shown on Fig. 6. The length of the fiber loops was 5131.7 $\mathrm{m}$ and $5177.7 \mathrm{~m}$. The length was chosen from the following considerations: the number of observed slots with respect to the coordinate $\mathrm{n}$ should be sufficiently large (about 200, as follows from Figure 1).

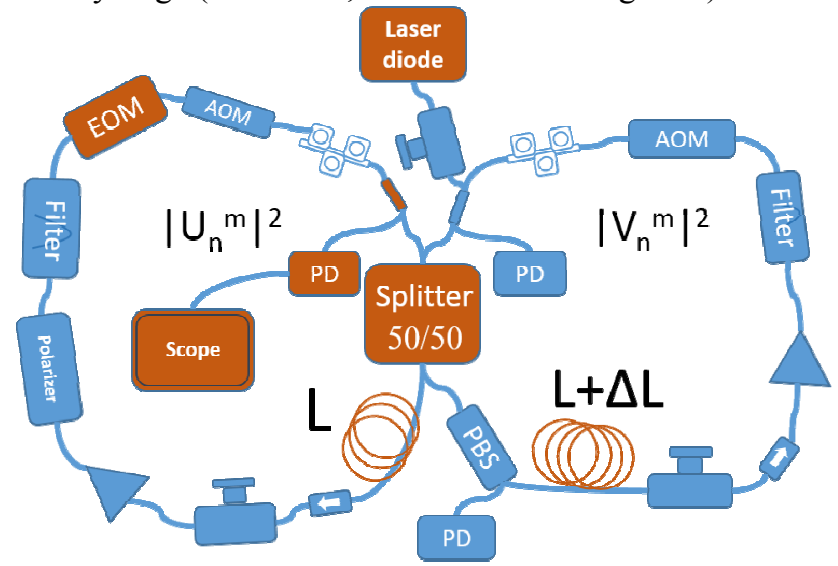

Figure 6. Experimental setup. 
TrueWave ${ }^{\circledR}$ REACH Fiber OFS fiber was used in the experiment. The pulse duration was chosen to be 100 nsec at a wavelength of $1550 \mathrm{~nm}$. To generate pulses a semiconductor laser diode with distributed feedback was used. The pulse power was of the order of 1 milliwatt. The limitation on the duration of the pulse was determined by the characteristics of the laser diode, since the laser pulse was created by direct modulation of the electrical current at the diode. The width of the spectrum of the laser pulse obtained was determined by the heterodyning method followed by Fourier analysis. The measurements showed that the width of the spectrum was $\sim 0.5 \mathrm{GHz}$.

The random potential was realized by means of a phase shift created by an electro-optical modulator with a sufficiently short response time operating at a frequency of up to $10 \mathrm{GHz}$.

To achieve the specified number off roundtrips (up to 200), it was necessary to control the polarization light pulses. The emission of a laser diode is polarized. But fibers used do not preserve polarization, while the semiconductor amplifiers and the electro-optical modulator are sensitive to polarization. The polarization was controlled with the help of polarization beam splitters. With the correct alignment of the polarization axes at all the elements, the polarization evolution of the pulses was reduced to a trivial evolution, i.e. at each point of the fiber, the polarization of the pulse did not change from roundtrip to roundtrip.

The next limiting factor in the experiment is noise. Since the losses were accurately compensated by those, there is a noise signal growth caused by the spontaneous emission of a laser diode and semiconductor amplifiers. The magnitude of the noise becomes significant with a large number of roundtrips and ultimately prevails over the useful signal. To overcome optical noise, the following method was chosen: the complete suppression of optical signals in loops with the help of acoustooptic modulators was made by increasing the losses in both loops by a large amount (about $13 \mathrm{~dB}$ ) for a long time before the start of the each experiment. Preliminary noise suppression allowed to increase the number of rounds with acceptable level of signal-to-noise ratio to 200. To further suppress spontaneous noise, optical spectral filters with adjustable width were put in both rings.

Another issue in the experimental observations with a large number of roundtrips is associated with the need to get rid of the influence of acoustic noises and temperature drifts. Indeed, the interference of pulses propagating along two loops will change significantly when the relative optical path in one of the loops changes by an amount of the order of the wavelength. In turn, an elongation by $1 \mu \mathrm{m}$ of a $5 \mathrm{~km}$ fiber spool occurs when the temperature of the entire fiber spool is simultaneously increased by only a hundredth of a degree, thus temperature drift does change the interference pattern. Then, the temperature drift leads to a change in the polarization properties of the fiber spools, that also changes the evolution of pulses. Finally, there is acoustic noise that leads to local stretching of the fibers and, accordingly, to a change of the optical paths. To protect the system, sound-absorbing and thermostabilized boxes were used.

Thus, in each experiment the system was cleaned from the noise of the amplifiers using acoustooptical modulators, then a single pulse from the semiconductor laser formed by direct modulation of the current was launched into the system,. The electro-optical modulator created additional phase shifts in one of the loops. Time dynamics of the chain of pulses at the output from one of the loops was registered by a digital oscilloscope. For each set of system parameters the experiment was repeated for 100 different realizations of randomness.

To demonstrate the localization effect, a picture of evolution was measured for various realizations of the optical potential with different levels of disorder $\varphi_{\max }$, varying from 0 to $\pi$. For each level of disorder, statistics for 100 different realizations was acquired. Further, the experimental dependences of the participation ratio (Fig.7), and the shape of the packet (Fig. 8) were found. They turned out to be similar to those calculated theoretically in the previous section. 


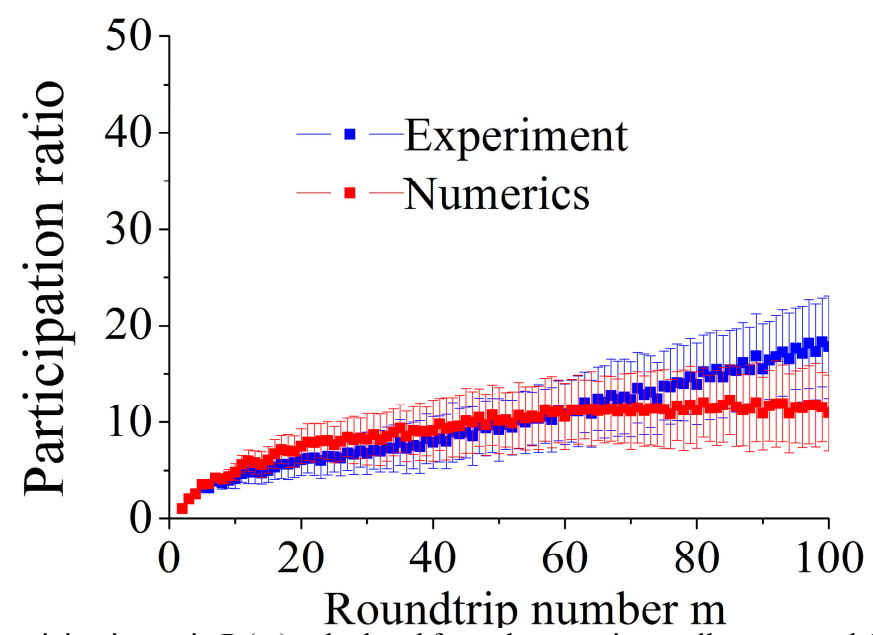

Figure 7. Dependence of the participation ratio $\mathrm{P}(\mathrm{m})$ calculated from the experimentally measured dependences of the amplitudes of the chain of pulses, and also by numerical simulation using the same realizations of the random potential of the strength of $0.8 \pi$. Experimental data are in good agreement with numerical calculations.

In complete agreement with numerical simulation, with increasing strength of disorder the degree of localization increases, and the participation ratio decreases. Though, the effect of noise begins to appear on $\mathrm{m} \sim 100$, and leads to an increase of the participation ratio.

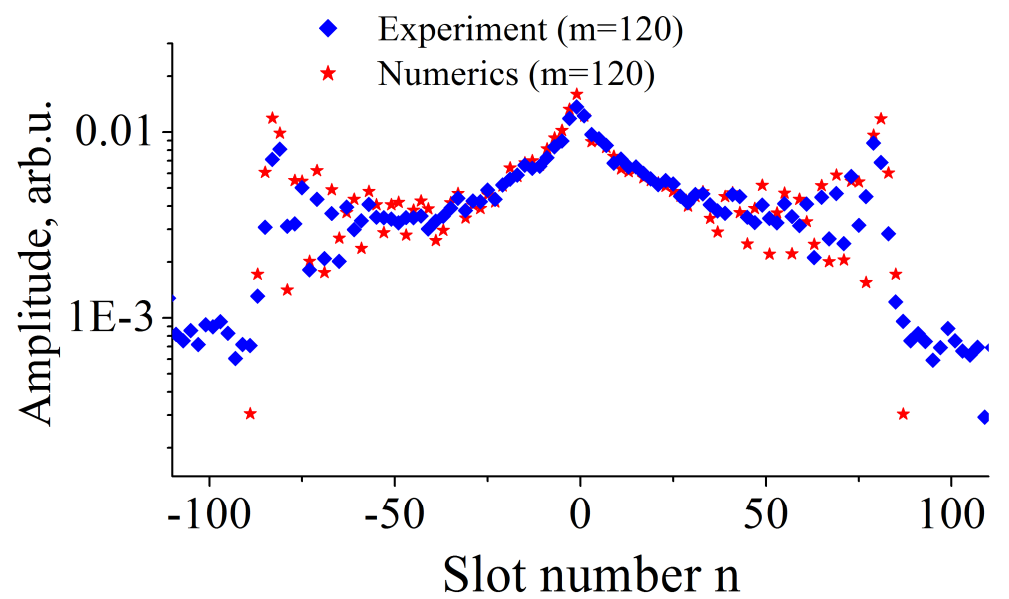

Figure 8. The distribution of the pulse intensity in the experiment (blue) and in the numerics (red) as a function of slot number $\mathrm{n}$ for the potential with $\varphi_{\max }=0.8 \pi$ and the number of rounds $\mathrm{m}=120$.

\section{CONCLUSION}

To conclude, we have simulated numerically and demonstrated experimentally Anderson localization in synthetic photonic mesh lattices. We find that whereas localized modes arise even for weak disorder in full agreement with the theory of Anderson localization in 1D systems, the strongest degree of localization is limited and increases in lattices with wider band-gaps.

This work is supported by the Russian Science Foundation (16-12-10402). A.A.S. acknowledges support by the Australian Research Council (ARC) (DP160100619). 


\section{REFERENCES}

[1] Regensburger, A., Bersch, C., Hinrichs, B., Onishchukov, G., Schreiber, A., Silberhorn, C.., Peschel, U., "Photon Propagation in a Discrete Fiber Network: An Interplay of Coherence and Losses," Phys. Rev. Lett. 107(23), $233902(2011)$.

[2] Regensburger, A., Miri, M.-A., Bersch, C., Näger, J., Onishchukov, G., Christodoulides, D. N.., Peschel, U., “Observation of Defect States in PT-Symmetric Optical Lattices," Phys. Rev. Lett. 110(22), 223902 (2013).

[3] Regensburger, A., Bersch, C., Miri, M., Onishchukov, G., Christodoulides, D. N.., Peschel, U., "Parity-time synthetic photonic lattices," Nature 488(7410), 167-171 (2012).

[4] Wimmer, M., Regensburger, A., Miri, M., Bersch, C., Christodoulides, D. N.., Peschel, U., "Observation of optical solitons in PT-symmetric lattices," Nat. Commun. 6, 7782 (2015).

[5] Anderson, P. W., “Absence of Diffusion in Certain Random Lattices," Phys. Rev. 109(5), 1492-1505 (1958).

[6] Lahini, Y., Avidan, A., Pozzi, F., Sorel, M., Morandotti, R., Christodoulides, D. N.., Silberberg, Y., "Anderson Localization and Nonlinearity in One-Dimensional Disordered Photonic Lattices," Phys. Rev. Lett. 100(1), 13906 (2008).

[7] Schwartz, T., Bartal, G., Fishman, S.., Segev, M., "Transport and Anderson localization in disordered two-dimensional photonic lattices," Nature 446(7131), 52-55 (2007).

[8] Sperling, T., B?hrer, W., Aegerter, C. M.., Maret, G., "Direct determination of the transition to localization of light in three dimensions," Nat. Photonics 7(1), 48-52 (2012).

[9] Karbasi, S., Frazier, R. J., Koch, K. W., Hawkins, T., Ballato, J.., Mafi, A., "Image transport through a disordered optical fibre mediated by transverse Anderson localization," Nat. Commun. 5, 1-9, Nature Publishing Group (2014).

[10] Ruocco, G., Abaie, B., Schirmacher, W., Mafi, A.., Leonetti, M., "Disorder-induced single-mode transmission," Nat. Commun. 8, 14571 (2017).

[11] Schreiber, A., Cassemiro, K. N., Potoček, V., Gábris, A., Jex, I.., Silberhorn, C., "Decoherence and Disorder in Quantum Walks: From Ballistic Spread to Localization," Phys. Rev. Lett. 106(18), 180403 (2011).

[12] Abrahams, E., Anderson, P. W., Licciardello, D. C.., Ramakrishnan, T. V., "Scaling Theory of Localization: Absence of Quantum Diffusion in Two Dimensions," Phys. Rev. Lett. 42(10), 673-676 (1979).

[13] Berezinskii, V. L., "Kinetics of a quantum particle in a one-dimensional random potential," Sov. Phys. JETP 38(3), 1251-1266 (1974).

[14] Pankov, A., Vatnik, I., Churkin, D. V.., Sukhorukov, A. A., "Surface waves in mesh synthetic photonic lattices," Proc. SPIE, 98851, 98851G (2016).

[15] Segev, M., Silberberg, Y.., Christodoulides, D. N., “Anderson localization of light,” Nat. Photonics 7(3), 197-204, Nature Publishing Group (2013). 